Chinese Journal of Organic Chemistry

NOTE

\title{
绿色合成抗癲㾋药氯西加酮及其类似物
}

\author{
刘改枝*, 吴 亚 ${ }^{a}$ 徐海伟 $b$ 刘宏民 $*, b$ \\ ( ${ }^{a}$ 河南中医学院药学院 郑州 450046) \\ ( ${ }^{b}$ 郑州大学药学院 郑州 450001)
}

\begin{abstract}
摘要 用温和高效的方法实现了抗癲㾋药氯西加酮及其系列类似物的非对映选择性绿色合成. 4-甲氧基呋喃酮与芳香 醛发生类似 Aldol 缩合的反应合成系列氯西加酮类似物. 通过结晶的方法高选择性地得到 $(d e \geqslant 96 \%)$ 氯西加酮. 在反应 体系中引入手性诱导试剂实现氯西加酮的非对映选择性合成. 所有新化合物的结构都经过 ${ }^{1} \mathrm{H} N M R,{ }^{13} \mathrm{C} N \mathrm{~N}$, IR, HRMS 确认.
\end{abstract}

关键词 绿色合成; 氯西加酮; 合成; 抗嘭痳药; 非对映选择性

\section{A Green Route for the Synthesis of Antiepileptic Drug Losigamone and Its Analogues}

\author{
Liu, Gaizhi*,a $\quad \mathrm{Wu}, \mathrm{Ya}^{a} \quad \mathrm{Xu}$, Haiwei $^{b} \quad$ Liu, Hongmin ${ }^{*, b}$ \\ ( ${ }^{a}$ Henan University of Traditional Chinese Medicine, Zhengzhou 450046) \\ ( ${ }^{b}$ School of Pharmaceutical Science, Zhengzhou University, Zhengzhou 450001)
}

\begin{abstract}
By an efficient and mild diastereoselective aldol-type reaction of aromatic aldehyde with 4-methoxyfuran2(5H)-one, losigamone and a series of its analogues were synthesized. Fortunately, the racemic threo-isomers of losigamone were obtained by crystallization with good de value $(\geqslant 96 \%)$. The introduction of chiral reagents to the reaction system resulted in diastereoselectivity to losigamone. All structures of new compounds were confirmed by ${ }^{1} \mathrm{H}$ NMR, ${ }^{13} \mathrm{C}$ NMR, IR and HRMS techniques.

Keywords green synthesis; losigamone; synthesis; anticonvulsive activity; diastereoselectivity
\end{abstract}

氯西加酮 (2a)(图 1) [AO-33; ( \pm$)-5(R, S)-\alpha(S, R)-$ 5-(2-氯芐基甲基)-4-甲氧基-2 $(5 H)$-呋喃酮], 是一种新型 的抗癫㾁药, 正在对部分性及继发性全身发作病人进行 三期临床试验 ${ }^{[1 \sim 3]}$. 氯西加酮抗惊厥活性的药学基团是 甲氧基取代的丁内酯环. 氯西加酮及其它合成的类似物 分子详细的构效关系研究表明, $\alpha$-羟芐基官能团也是必 不可少的活性基团. 氯西加酮及其相关类似物的赤式异 构体没有药理活性或者与相应的苏式异构体相比活性 比较差 ${ }^{[4]}$. 许多文献报道了氯西加酮的合成 ${ }^{[5>7]}$, 但是 大多数合成方法需要用到金属试剂或者反应条件比较 苛刻. 在笛选氯西加酮所做的系统构效研究中没有考虑 到分子中 4-拜乙酰乙酸内酯部分的重要性. 研究表明, 利用电子等排原理将某些化合物内酯部分替换为硫代 内酯表现出来相似的或者更好的药理活性, 例如 thiopilocarpin ${ }^{[8]}$, thiobasidalin ${ }^{[9]}$ 和 thioascorbic acid ${ }^{[10]}$. 因 此, Schachtner 等和他的合作者 ${ }^{[11]}$ 报道了利用 “生物等排 性原理” 合成的氯西加酮类似物 thiolosigamone (4). 很 不幸的是, 与氯西加酮相比, thiolosigamone (4) 只有在 高浓度下才表现出非常小的抗癫㾋活性.

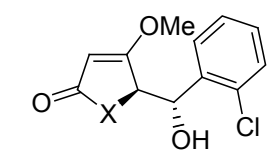

$$
\begin{aligned}
& X=\mathrm{O} \text { : Losigamone } \mathbf{2 a} \text { (rac.) } \\
& X=\mathrm{S} \text { : Thiolosigamone } 4 \text { (rac.) }
\end{aligned}
$$

图 1 化合物 $2 \mathrm{a}$ 和 4 的结构

Figure 1 Structures of compounds 2a and $\mathbf{4}$

我们实验室发现了一种温和的高收率非对映选择

\footnotetext{
*E-mail: liugaizhi@126.com; liuhm@zzu.edu.cn

Received May 3, 2013; revised May 24, 2013; published online June 4, 2013.

Project supported by the Doctoral Research Foundation of Henan University of Traditional Chinese Medicine (No. BSJJ2010-03).

河南中医学院博士基金(No. BSJJ2010-03)资助项目.
} 
性地合成氯西加酮的新方法. 根据氯西加酩的结构特 点, 结合我们 ${ }^{[12 ~ 16]}$ 前期对丁烯内酯的研究工作, 本文阐 述了氯西加酮及其系列类似物的绿色合成.

\section{1 结果与讨论}

基于前期对 $\gamma$ 丁烯内酯类化合物的合成研究, 邻氯 苯甲醛直接与 4-甲氧基呋喃酩(1)反应, 磷酸盐(PBS)缓 冲液为溶剂, 加入催化量的吐温 80 , 弱碱无水碳酸钠为 催化剂(Eq. 1). 室温反应 $6 \mathrm{~h}$ 薄层检测至反应完全, 得 $1: 1$ 的非对映异构体 2 和 3 的混合物. 氯西加酮的合成 过程中, 从未经分离的产物 ${ }^{1} \mathrm{H}$ NMR 图谱上可以看出, 在 $\delta 3.9$ 和 3.7 处分别对应一个 $\mathrm{OCH}_{3}$ 的峰, 分别对应一 对赤式异构体 3a 和一对苏式异构体 2a, 两对非对映异 构体比例为 $1: 1$. 用乙酸乙酯对产物进行重结晶纯化 后所得结晶产物的 ${ }^{1} \mathrm{H}$ NMR 图谱显示只在 $\delta 3.7$ 处有一 个 $\mathrm{OCH}_{3}$ 峰, 即一对苏式异构体氯西加酮 $(\mathbf{2 a})$. 同理, 其 他氯西加酮的类似物可以用相似的方法分离纯化.

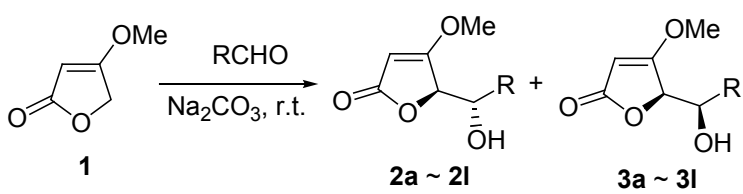

结晶所得氯西加酮及其类似物的比例可以通过 HPLC 分析得知. 非对映异构体 $\mathbf{2}$ 和 $\mathbf{3}$ 通过乙酸乙酯结 晶的方法很容易分离. 结晶所得氯西加酮(2a) 的 HPLC 图显示, 初次结晶后苏式异构体的比例高达 $96 \%$ 以上, 即氯西加酮(2a)的 $d e$ 值约 96\%(图 2). 通过多次结晶的 方法可以进一步提高产物氯西加酮的纯度. 同理, 氯西 加酮的系列类似物通过上述方法, 4-甲氧基呋喃醖(1)与 不同的芳香醛反应制得(表 1). 将系列类似物直接用乙 酸乙酯进行初次结晶所得产物的 $d e$ 值及收率如表 1 所 示.

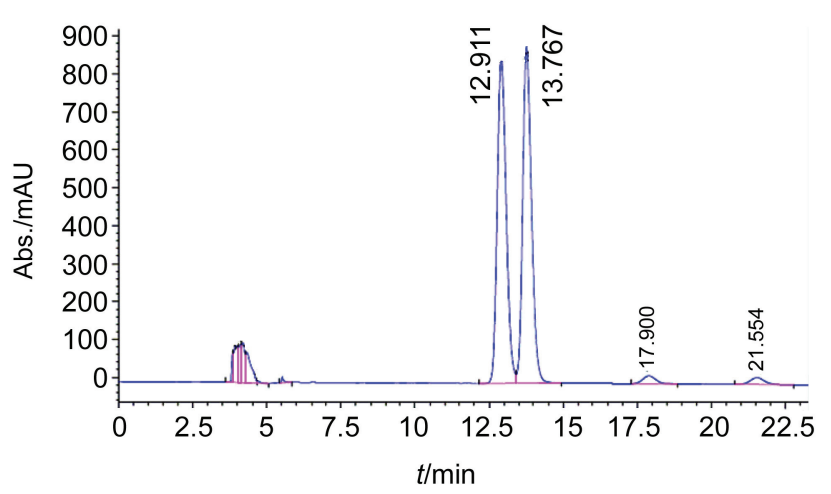

图 2 氯西加酮 (2a) 的 HPLC 图

Figure 2 The HPLC spectrum of 2a (losigamone)

结晶产物所得化合物 2a 即氯西加酮为一对苏式异 构体, 其绝对构型通过 X 单晶衍射分析得到确证. 由于
分子间氢键作用，外消旋的氯西加酮以孪晶的形式结晶 析出(图 3 所示). 与氯西加酮类似，化合物 $\mathbf{1}$ 与不同的芳 香醛反应得系列氯西加酮类似物(表 1 所示). 系列类似 物的结构通过 NMR, IR, MS 等确证, 他们的 $d e$ 值通过 ${ }^{1} \mathrm{H}$ NMR 或者 HPLC 分析可知. 大多数类似物可以通过 结晶的方法得到分离纯化，他们的苏式异构体同样是以 孪晶的形式从母液中结晶析出, 通过重结晶的方式可以 提高非对映选择性 $d e$ 值.
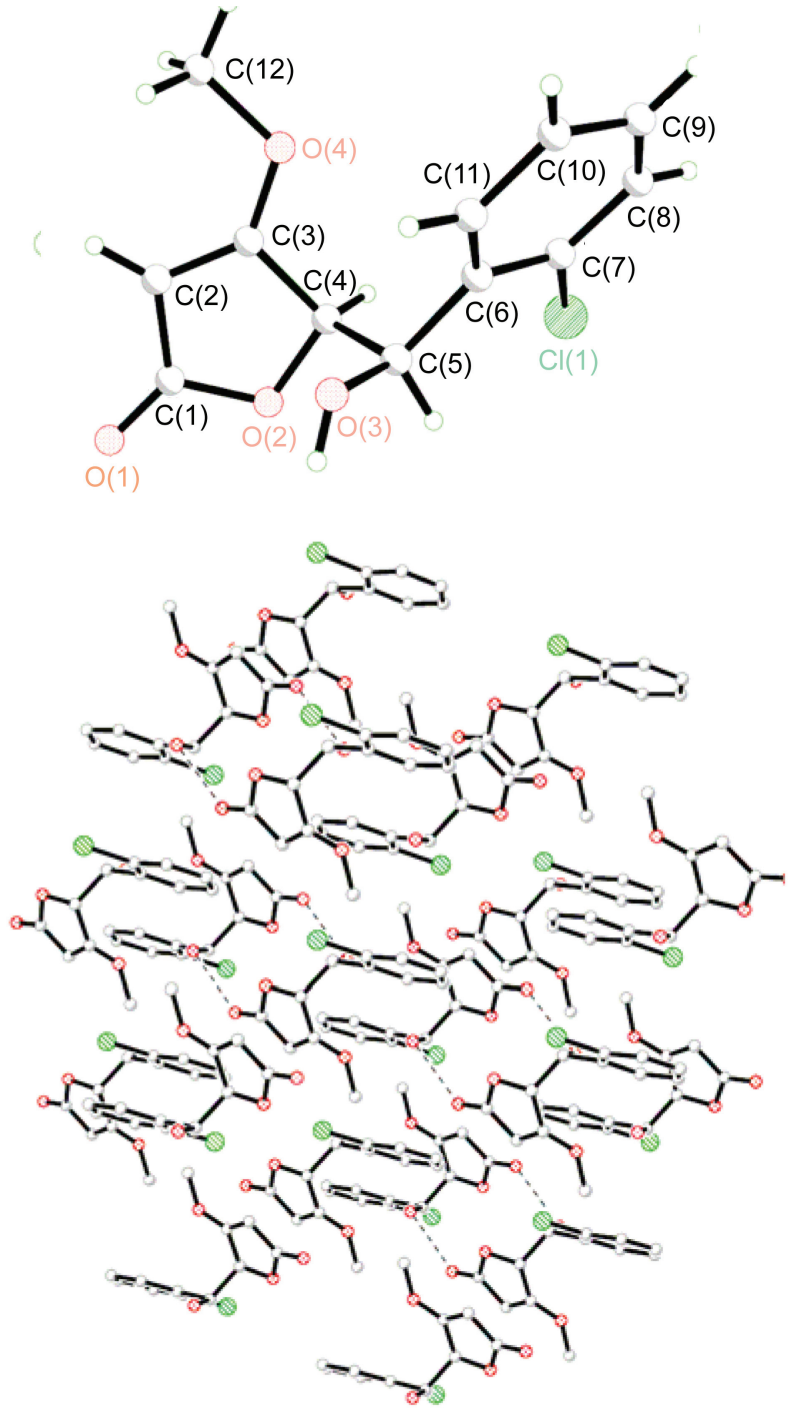

图 3 氯西加酮(2a)的 X 单晶衍射图和堆积图

Figure 3 The X-ray structure and packing diagram of 2a (losigamone)

从结晶产物 2a 和 $2 \mathbf{c}$ (图 4)的单晶衍射图可知, 所得 产物为一对外消旋苏式异构体. 一对旋光异构体 $R S$ 和 $S R$ 通过分子间氢键的作用以孪晶的形式结晶, 另一对 异构体 $R R$ 和 $S S$ 无法形成类似的孪晶结构. 因此，这两 种非对映异构体表现出了不同的溶解性，所以可以通过 结晶的方式得到很好的分离纯化.

用 HPLC 对反应体系的检测可知, 氯西加酮(2a)及 
表 1 合成的化合物

Table 1 Compounds synthesized

化合物编号

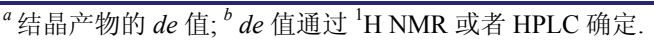

其非对映异构体 3a 以 $1: 1$ 的比例生成, 为了提高氯西 加酮的收率, 我们考察了通过向反应体系中引入手性试 剂的方法来提高反应的非对映选择性. 现在我们试图弄 清并证实是否手性试剂的引入可以得到氯西加酮选择 性过量的产物. 我们主要考察了不同手性试剂的引入对 化合物 1 与邻氯苯甲醛反应的影响, 产物结构见 Scheme 1 , 结果见表 2 所示. 结果显示, 手性试剂的引入对氯西 加酮非对映选择性的提高有一定作用.

表 2 不同手性试剂诱导下氯西加酮的 $d e$ 值 $^{a}$

Table 2 The $d e$ values of losigamone by different chiral source induced

\begin{tabular}{ccccc}
\hline 手性试剂 & $\beta-\mathrm{CD}$ & 葡萄糖 & $R R$ 氨基醇 & $S S$ 氨基醇 \\
\hline$d e$ 值 & 37 & 39 & 38 & 50 \\
\hline
\end{tabular}

${ }^{a} \mathrm{HPLC}$ 检测的反应体系的 $d e$ 值.

\section{2 结论}

研究了一种简单高效的绿色合成方法. 高收率合成 了抗癫戍药氯西加酮及其系列类似物. 通过结晶的方法 得到 $d e$ 值高达 $96 \%$ 的氯西加酮产物, 通过重结晶的方<smiles>O=C(O)CNC[C@H](O)COc1ccccc1</smiles><smiles>OC(CNCC(O)COc1ccccc1)COc1ccccc1</smiles>

Scheme 1

法可以进一步提高氯西加酮的纯度. 系列氯西加酮类似 物同样可以用结晶的方式得到. 并考察了手性试剂的引 入对氯西加酮非对映选择性合成的影响，通过不同手性 试剂的引入, 可以有效提高氯西加酮的非对映选择性合 成收率.

\section{3 实验部分}

\section{1 仪器与试剂}

所有试剂和溶剂从化学试剂供应商购得. 所有反应 通过 TLC 或者 HPLC 检测. 熔点使用四川大学科仪厂生 


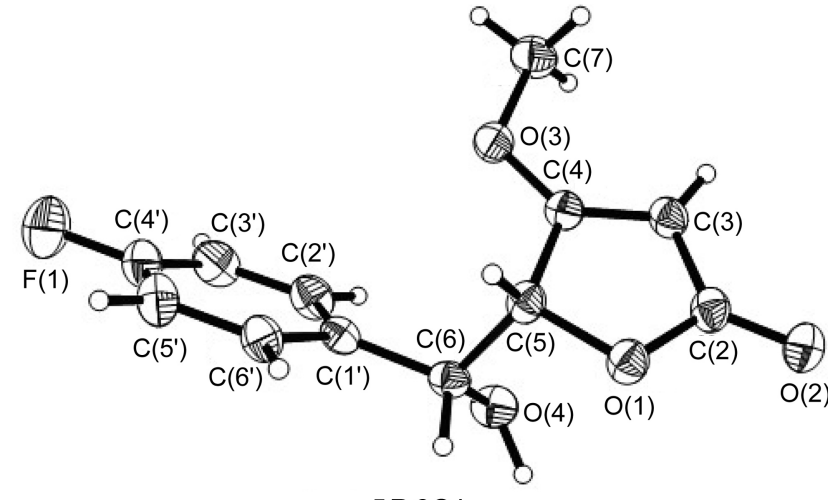

$5 R, 6 \mathrm{~S}$-isomer

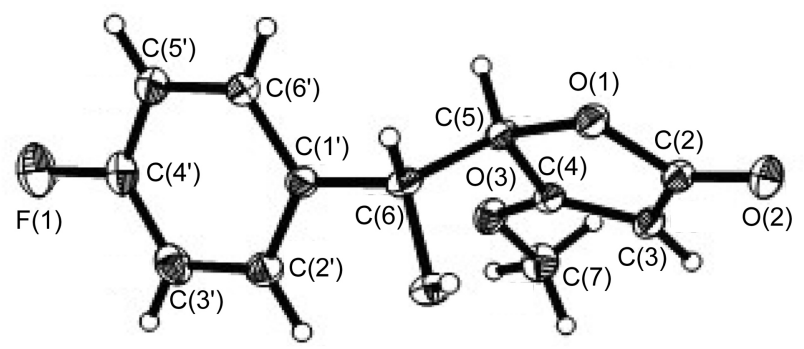

$5 R, 6 S$-isomer

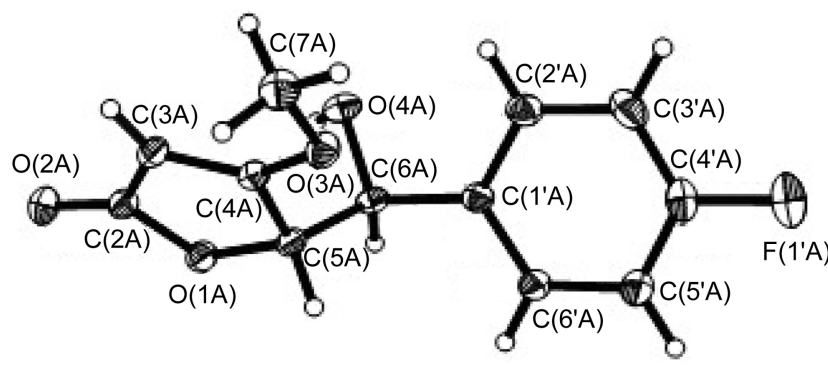

$5 S, 6 R$-isomer

图 4 化合物 $2 \mathrm{c}$ 的 X 单晶衍射图

Figure 4 X-ray structure of compound $2 c$

产的 WC-1 型显微熔点仪测定, 温度计未经校正; IR 使 用西德 PE 公司产 PE-1710 型红外分光光度计测定, $\mathrm{KBr}$ 压片; ${ }^{1} \mathrm{H}$ NMR, ${ }^{13} \mathrm{C}$ NMR 和 2D NMR 使用瑞典 Bruker DPX-400 型超导核磁共振仪测定; 高分辨质谱使用 ESI 技术用 Waters Micromass 公司 Q-Tof MicoTM 质谱仪测 定; X 单晶衍射使用 Rigaku RAXIS-IV 测得.

\section{2 实验方法}

氯西加酮及其类似物合成通法: 化合物 1 (100 mg, $0.87 \mathrm{mmol})$ 和无水碳酸钠 $(0.01 \mathrm{~mol})$ 溶于 PBS 缓冲液中, 然后加入不同的芳香醛 $(0.012 \mathrm{~mol})$. 混合物室温摚拌 $3 \sim 12 \mathrm{~h}$, TLC 检测至反应完全. 反应混合物分别用水 $(30 \mathrm{~mL} \times 2)$ 和乙酸乙酯 $(50 \mathrm{~mL} \times 2)$ 洗, 有机层用无水硫 酸钠干燥结晶得氯西加酮及系列类似物.

(土)苏式-5-[(2-氯苯基-羟甲基)-4-甲氧基- $5 \mathrm{H}$-呋喃2-酮(2a): 白色结晶, 产率 95\%. m.p. 89 $90{ }^{\circ} \mathrm{C} ;{ }^{1} \mathrm{H}$ NMR $\left(400 \mathrm{MHz}, \mathrm{CDCl}_{3}\right) \delta: 7.61 \sim 7.64(\mathrm{~m}, 1 \mathrm{H}), 7.31 \sim$ $7.36(\mathrm{~m}, 1 \mathrm{H}), 7.32 \sim 7.34(\mathrm{~m}, 1 \mathrm{H}), 7.24 \sim 7.27(\mathrm{~m}, 1 \mathrm{H})$, $5.56 \sim 5.59(\mathrm{~m}, 1 \mathrm{H}), 5.18 \sim 5.23$ (overlap, $1 \mathrm{H}$ ), $5.08(\mathrm{~s}$, 1H), 3.75 (s, 3H), $2.5 \sim 3.5$ (overlap, $1 \mathrm{H}) ;{ }^{13} \mathrm{C}$ NMR $(100.6$ $\left.\mathrm{MHz}, \mathrm{CDCl}_{3}\right) \delta: 179.8,172.3,134.9,131.9,129.4,129.2$, 90.4, 80.3, 77.2, 70.0, 59.3; IR (KBr) v: 3368, 1736, 1628 $\mathrm{cm}^{-1}$; HR-MS (ESI) calcd for $\mathrm{C}_{12} \mathrm{H}_{12} \mathrm{ClO}_{4}[\mathrm{M}+\mathrm{H}]^{+}$ 255.0424 , found 255.0419 .

（土）苏式-5-(羟苯甲基)-4-甲氧基-5 $\mathrm{H}$-呋喃-2-酮 (2b): 白色结晶, 产率 $90 \%$. m.p. 101 102 ${ }^{\circ} \mathrm{C} ;{ }^{1} \mathrm{H}$ NMR $\left(400 \mathrm{MHz}, \mathrm{CDCl}_{3}\right) \delta: 7.42 \sim 7.46(\mathrm{~m}, 2 \mathrm{H}), 7.37 \sim 7.39(\mathrm{~m}$, $2 \mathrm{H}), 7.24 \sim 7.27(\mathrm{~m}, 1 \mathrm{H}), 5.11(\mathrm{~s}, 1 \mathrm{H}), 4.96 \sim 5.00$ (overlap, $2 \mathrm{H}$ ), 3.9 (s, 3H), 2.46 2.50 (overlap, $1 \mathrm{H}$ ); ${ }^{13} \mathrm{C}$ NMR $\left(100.6 \mathrm{MHz}, \mathrm{CDCl}_{3}\right) \delta: 179.8,172.3,138.9,128.6$, 126.6, 90.1, 81.3, 77.0, 72.2, 59.6; IR (KBr) v: 3404, 1751, $1722,1628 \mathrm{~cm}^{-1}$; HR-MS (ESI) calcd for $\mathrm{C}_{12} \mathrm{H}_{13} \mathrm{O}_{4}[\mathrm{M}+$ $\mathrm{H}^{+}$221.0814, found 221.0811.

(土) 苏式-5-[(4-氟苯基)-羟甲基]-4-甲氧基-5H-呋 喃-2-酮(2c): 白色结晶, 产率 93\%. m.p. 80 $81{ }^{\circ} \mathrm{C} ;{ }^{1} \mathrm{H}$ NMR (400 MHz, $\left.\mathrm{CDCl}_{3}\right) \delta: 7.41 \sim 7.43(\mathrm{~m}, 2 \mathrm{H}), 7.04 \sim$ $7.06(\mathrm{~m}, 2 \mathrm{H}), 5.11(\mathrm{~s}, 1 \mathrm{H}), 4.93 \sim 4.97(\mathrm{~m}, 1 \mathrm{H}), 4.91 \sim$ $4.93(\mathrm{~m}, 1 \mathrm{H}), 3.90$ (s, 3H), $2.4 \sim 3.0$ (overlap, $1 \mathrm{H}$ ); ${ }^{13} \mathrm{C}$ NMR $\left(100.6 \mathrm{MHz}, \mathrm{CDCl}_{3}\right) \delta: 179.7,172.3,134.8,128.5$, $115.5,90.3,81.3,77.2,71.7,59.5$; IR (KBr) $v: 3333,1719$, $1634,1513 \mathrm{~cm}^{-1}$; HR-MS (ESI) calcd for $\mathrm{C}_{12} \mathrm{H}_{12} \mathrm{FO}_{4}[\mathrm{M}+$ $\mathrm{H}]^{+}$239.0720, found 239.0735.

(土)苏式-5-[(3,4-二甲氧基苯基)-羟甲基]-4-甲氧基5H-呋喃-2-酮(2d): 白色结晶, 产率 91\%. m.p. 78 79 ${ }^{\circ} \mathrm{C} ;{ }^{1} \mathrm{H}$ NMR $\left(400 \mathrm{MHz}, \mathrm{CDCl}_{3}\right) \delta: 6.96(\mathrm{~s}, 1 \mathrm{H}), 6.87 \sim$ $6.90(\mathrm{~m}, 1 \mathrm{H}), 6.79(\mathrm{~d}, J=8.0 \mathrm{~Hz}, 1 \mathrm{H}), 5.97(\mathrm{~s}, 2 \mathrm{H}), 5.12$ (s, 1H), 4.86 4.91 (overlap, 2H), 3.91 (s, 3H); ${ }^{13} \mathrm{C}$ NMR $\left(100.6 \mathrm{MHz}, \mathrm{CDCl}_{3}\right) \delta: 179.7,172.2,147.8,132.8,120.4$, 108.2, 107.3, 101.2, 90.1, 81.3, 77.2, 72.2, 59.6; IR (KBr) $v: 3318,1722,1650 \mathrm{~cm}^{-1}$; HR-MS (ESI) calcd for $\mathrm{C}_{13} \mathrm{H}_{13} \mathrm{O}_{6}[\mathrm{M}+\mathrm{H}]^{+}$265.0712, found 265.0711.

5-[(4-氯苯基)-羟甲基]-4-甲氧基- $5 \mathrm{H}$-呋喃-2-酮 (2e): 白色结晶, 产率 96\%. m.p. 87 $88{ }^{\circ} \mathrm{C} ;{ }^{1} \mathrm{H}$ NMR $\left(400 \mathrm{MHz}, \mathrm{CDCl}_{3}\right) \delta: 7.35 \sim 7.37(\mathrm{~m}, 2 \mathrm{H}), 7.24 \sim 7.24(\mathrm{~m}$, $2 \mathrm{H}), 5.10(\mathrm{~s}, 1 \mathrm{H}), 5.05(\mathrm{~s}, 1 \mathrm{H}), 4.93 \sim 4.97(\mathrm{~m}, 1 \mathrm{H}), 4.93$ (s, 1H), $3.90(\mathrm{~s}, 3 \mathrm{H}), 3.79$ (s, $1.75 \mathrm{H}) ;{ }^{13} \mathrm{C}$ NMR $(100.6$ $\left.\mathrm{MHz}, \mathrm{CDCl}_{3}\right) \delta: 179.5,172.3,137.5,135.6,134.3,115.5$, 90.4, 81.3, 73.0, 71.6, 59.5. IR (KBr) v: 3384, 1751, 1710, $1627 \mathrm{~cm}^{-1}$; HR-MS (ESI) calcd for $\mathrm{C}_{12} \mathrm{H}_{12} \mathrm{ClO}_{4}[\mathrm{M}+\mathrm{H}]^{+}$ 255.0424, found 255.0439.

5-[(2-甲氧基苯基)-羟甲基]-4-甲氧基- $5 \mathrm{H}$-呋喃-2-酮 (2f): 色结晶, 产率 $95 \%$. m.p. $109 \sim 110{ }^{\circ} \mathrm{C} ;{ }^{1} \mathrm{H}$ NMR 
$\left(400 \mathrm{MHz}, \mathrm{CDCl}_{3}\right) \delta: 7.43(\mathrm{~d}, J=8.0 \mathrm{~Hz}, 1 \mathrm{H}), 7.30 \sim 7.33$ $(\mathrm{m}, 1 \mathrm{H}), 6.98 \sim 7.01(\mathrm{~m}, 1 \mathrm{H}), 6.90(\mathrm{~d}, J=8.0 \mathrm{~Hz}, 1 \mathrm{H})$, $5.30(\mathrm{~s}, 1 \mathrm{H}), 5.20(\mathrm{~s}, 1 \mathrm{H}), 5.03(\mathrm{~d}, J=1.7 \mathrm{~Hz}, 1 \mathrm{H}), 3.92$ (s, $3 \mathrm{H}), 3.87$ (s, 3H), $2.3 \sim 3.2$ (overlap, $1 \mathrm{H}$ ); ${ }^{13} \mathrm{C}$ NMR (100.6 $\left.\mathrm{MHz}, \mathrm{CDCl}_{3}\right) \delta: 179.5,172.3,137.5,135.6,134.3,115.5$, 90.4, 81.3, 73.0, 71.6, 59.5; IR (KBr) v: 3360, 1730, 1628 $\mathrm{cm}^{-1}$; HR-MS (ESI) calcd for $\mathrm{C}_{13} \mathrm{H}_{15} \mathrm{O}_{5}[\mathrm{M}+\mathrm{H}]^{+}$ 251.0920, found 251.0926.

苏式-5-(1-羊基-3-苯丙烯基)-4-甲氧基-5H-㫙喃-2酥(2g): 白色结晶, 产率 89\%. m.p. $111 \sim 112{ }^{\circ} \mathrm{C} ;{ }^{1} \mathrm{H}$ NMR $\left(400 \mathrm{MHz}, \mathrm{CDCl}_{3}\right) \delta: 7.23 \sim 7.38(\mathrm{~m}, 10 \mathrm{H}), 6.62 \sim$ $6.73(\mathrm{~m}, 2 \mathrm{H}), 6.34(\mathrm{dd}, J=6.6,15.9 \mathrm{~Hz}, 1 \mathrm{H}), 6.19(\mathrm{dd}, J=$ $6.6,15.9 \mathrm{~Hz}, 1 \mathrm{H}), 5.14(\mathrm{~s}, 1 \mathrm{H}), 5.10(\mathrm{~s}, 1 \mathrm{H}), 4.91$ (d, $J=$ $1.7 \mathrm{~Hz}, 1 \mathrm{H}), 3.87$ (s, 3H), 3.82 (s, 3H), 2.8 3.3 (overlap, $1 \mathrm{H}) ;{ }^{13} \mathrm{C}$ NMR $\left(100.6 \mathrm{MHz}, \mathrm{CDCl}_{3}\right) \delta: 180.0,173.1,136.2$, $133.5,133.1,128.7,128.1,126.7,124.6,90.3,90.1,81.8$, 81.5, 72.3, 70.8, 59.7; IR (KBr) v: 3404, 1753, $1627 \mathrm{~cm}^{-1}$; HR-MS (ESI) calcd for $\mathrm{C}_{14} \mathrm{H}_{15} \mathrm{O}_{4}[\mathrm{M}+\mathrm{H}]^{+}$247.0970, found 247.0955 .

\section{References}

[1] Chatterjee, S. S.; Noldner, M. CNS Drug Rev. 1997, 3, 225.

[2] Borowicz, K. K.; Jaszczyk, B.; Luszczki, J. J.; Czuczwar, S. J. Eur. J. Pharmacol. 2007, 567, 110.

[3] Borowicz, K. K.; Kimber-Trojnar, Z.; Ratnaraj, N.; Patsalos, P. N.;
Luszczki, J. J.; Czuczwar, S. J. Eur. Neuropsychopharm. 2007, 17, 94.

[4] Kleuing, K.; Chatterjee, S. S. Abstract Book, International Symposium on "Dridimensionale Struktur und Wirkung", Schliersee, Germany, October $2 \sim 4,1989$.

[5] Laffan, D. US 5200531, 1993 [Chem. Abstr. 1993, 117, 131051].

[6] Antane, S.; Caufield, C. E.; Hu, W.; Keeney, D.; Labthavikul, P.; Morris, K.; Naughton, S. M.; Petersen, P. J.; Rasmussen, B. A.; Singh, G.; Yang, Y. J. Bioorg. Med. Chem. Lett. 2006, 16, 176.

[7] Pelter, A.; Al-Bayati, R. I. H.; Ayoub, M. T.; Lewis, W.; Pardasani, P.; Hansel, R. J. Chem. Soc., Perkin Trans. 1 1987, 717.

[8] Shapiro, G.; Floersheim, P.; Boelsterli, J.; Anstutz, R.; Bolligcr, G.; Mmenthaler, H.; Gmelin, G.; Suwwilai, P.; Walkinshaw, M. J. Med. Chem. 1992, 35, 15.

[9] Schachtner, J.; Stachel, H.-D.; Polborn, K.; Anke, T. Eur. J. Med. Chem. 1998, 33, 309.

[10] Stachel, H.-D.; Schxhtner, J.; Lotter, H. Tetrahedron 1993, 49, 4871.

[11] Josef, E. S.; Stachel, H. D.; Chatterjee, S. S.; Kurt Polborn, H. H. Eur. J. Med. Chem. 1998, 33, 665.

[12] Xu, H. W.; Wang, J. F.; Liu, G. Z.; Hong, G. F.; Liu, H. M. Org. Biomol. Chem. 2007, 5, 1247.

[13] Xu, H. W.; Dai, G. F.; Liu, G. Z.; Wang, J. F.; Liu, H. M. Bioorg. Med. Chem. 2007, 15, 4247.

[14] Xu, H. W.; Liu, G. Z.; Dai, G. F.; Wu, C. L.; Liu, H. M. Drug Discoveries Ther. 2007, 1(1), 73.

[15] Dai, G. F.; Xu, H. W.; Wang, J. F.; Liu, F. W. Liu, H. M. Bioorg. Med. Chem. Lett. 2006, 16, 2710.

[16] Liu, G. Z.; Han, D. N.; Wang, P.; Liu, H. M. Chin. J. Org. Chem. 2013, 33, DOI: 10.6023/cjoc201212050 (in Chinese). (刘改枝, 韩德恩, 王鹏, 刘宏民, 有机化学, 2013, 33, DOI: 10.6023/cjoc201212050.) 